\title{
Las TIC, factor de efectividad en la aplicación del mercadeo digital en negocios de retail
}

\begin{abstract}
FECHA DE RECEPCIÓN: 17 de enero FECHA DE APROBACIÓN: 1 de febrero Pp. 105-116

\section{Resumen}

La necesidad de desarrollo organizacional ha llevado a las empresas a repensar sus negocios, y a buscar la forma de rediseñarse en muchos aspectos para enfrentar la competencia y sostenerse en el mercado. El rápido crecimiento de las organizaciones se acompaña con la ampliación de su estructura funcional, sumado al incremento de volumen en sus actividades. En la medida en que cambian las situaciones, las empresas se transforman para asimilar con prontitud, los nuevos retos. El mercadeo digital constituye, hoy en día, una alternativa importante para el desenvolvimiento de las ventas. Esta vivencia de crecimiento y de cambio se apoya sustancialmente en las tecnologías de la información -TIC-, desempeñando un rol fundamental en el camino de la supervivencia, la generación de ventajas competitivas y un mayor valor añadido, al reducir cuellos de botella y restricciones en sus procesos, siempre que se empleen las herramientas adecuadas y se establezcan sinergias coincidentes entre usuarios y tecnología, de tal forma, que no sean relaciones de apropiación, sino de representación.

\section{Palabras clave}

Mercadeo digital, tecnologías de información, ventajas competitivas, valor añadido.

\section{Ricardo Prada Ospina}

Doctor en Ciencias Empresariales de la Universidad Antonio de Nebrija, -España-y doctor en Gestión de la Universidad EAN. MBA - Magíster en Administración. Especialista en Ingeniería de Producción. Ingeniero Mecánico. Profesor Asociado, Facultad de Administración Finanzas y Ciencias Economicas. 


\title{
ICT, an Effectiveness Factor in the Application of Digital Marketing to Retail Businesses
}

\begin{abstract}
Abstrac. The need for organizational development has led companies to review their business model and to find the way to redesign it to face the existing competition and to remain sustainable in the market. The rapid growth of organizations is accompanied by an increase of their functions, as well as an increase in their amount of activities. As situations change, companies are transformed to face new challenges almost instantly. Currently, digital marketing seems to be an important alternative for performing their sales. This evidence of fast increase and change is supported by ICT new technologies, performing a key role on the way to survival, the generation of competitive advantages and much more added value to avoid bottlenecks and restrictions in their processes unless they use appropriate tools and set up coincidental synergies between customers and new technologies so that they won't turn into appropriation relations better than into representation relations.
\end{abstract}

Key words. Digital marketing, ICT, competetive advantages, added value.

\section{Les TIC : facteurs d'efficacité lors de la mise en place du marketing digital dans la vente au détail}

Resumé. Le besoin de développement organisationnel a amené les entreprises à repenser leurs activités et à rechercher le moyen de se renouveler sur différents aspects pour faire face à la concurrence et se maintenir sur les marchés. La croissance rapide des organisations s'accompagne d'une croissance de leur structure fonctionnelle ainsi que de la hausse du volume de leurs activités. Dans la mesure où l'environnement des entreprises change, celles-cise transforment rapidement pour relever les nouveaux défis. La commercialisation en ligne constitue de nos jours une alternative importante pour l'augmentation des ventes. Cette expérience de croissance et de changement s'appuie de manière substantielle sur les technologies de l'information TIC - qui jouent un rôle fondamental pour la survie de l'entreprise, la création d'avantages compétitifs et la hausse de valeur ajoutée en réduisant certains goulots d'étranglement et restrictions des processus lorsque les outils adéquats sont employés et que des synergies concordantes entre les utilisateurs et la technologie s'établissent, de telle façon qu' il ne s'agit pas de relations d'appropriation mais de représentation.

Mots clefs. Commercialisation digitale, technologies de l'information, avantages compétitifs, valeur ajoutée.

\section{As TICs, fator de eficácia na implementação de Marketing Digital em negócios de Varejo}

Resumo. A necessidade de desenvolvimento organizacional tem levado as empresas a repensar seus negócios, e a buscar formas de redesenhar-se em muitos aspectos para enfrentar da melhor maneira a concorrência e manter-se no mercado. O rápido crescimento das organizações é acompanhado pela expansão da sua estrutura funcional, juntamente com o aumento do volume de suas atividades. Na medida em que as situações mudam, as empresas se transformam para assimilar prontamente novos desafios. O marketing digital é hoje, uma alternativa importante no desenvolvimento das vendas. Esta experiência de crescimento e mudança é baseada substancialmente em tecnologia da informação com as TICS, desempenhando um papel fundamental na forma de sobrevivência, na geração de vantagens competitivas e um maior valor agregado, ao reduzir dificuldades e restrições em seus processos, desde que as ferramentas apropriadas sejam usadas e se estabeleça sinergia entre os usuários e a tecnologia, de modo que não sejam relações de propriedade, mas de representação.

Palabras chave. Marketing digital, tecnologia da informação, vantagens competitivas, valor agregado. 


\section{Introducción}

T ntre más consensuado, estructurado y planificado sea el trabajo de la organización, se presentan menos inconvenientes, aprovechando mejor la experiencia de las personas de la organización. Este conocimiento, crece en la medida en que se establezca un diálogo abierto y constante entre las personas, en busca de soluciones y resultados con base en dicho conocimiento.

\section{El problema}

M ecanizados y Eléctricos S.A. y su retail $^{2}$ es una institución con más de medio siglo de experiencia y evolución dentro del mercado local. Representa un amplio concepto para la satisfacción de las necesidades de los clientes en diversas áreas, tales como: hogar, decoración, muebles, iluminación, electrónica, electrodomésticos, telefonía, seguridad, ferretería, enfriamiento y calefacción; acabados de construcción, equipos y motores; eléctricos, automotriz, finca y jardín; deportes, mascotas, BBQ, camping, cuidado personal, juguetería y minimarket, entre otros; donde se manejan más de 18.000 productos con 26.000 referencias diferentes, de las cuales el $80 \%$ de ellas están basadas en productos importados, y el otro $20 \%$ en productos nacionales.

La empresa Mecanizados y Eléctricos S.A. basa su esquema de negocio en el campo de grandes superficies, con un total
El presente artículo pretende interpretar el rol de las TIC en las organizaciones, y para ello, aporta el caso del grupo empresarial Mecanizados y Eléctricos S.A. ${ }^{1}$ y su filial como retail, a través de la descripción de la situación actual de tecnología en dicha compañía y de cómo las TIC, ante una necesidad inmediata, permiten, mediante el análisis y diseño de nuevas estrategias y procesos corporativos, integrar los sistemas de información para alcanzar la estructura más apropiada, con la tecnología más adecuada. de seis sucursales y alrededor de 300 puestos de trabajo distribuidos principalmente en su razón comercial como retail.

Al pertenecer al segmento de grandes superficies, el núcleo de negocio se concentra en las ventas que se realizan diariamente en cada una de sus sucursales, las cuales se encuentran conectadas directamente con la sede principal a través de los sistemas de facturación y logística centrales. Así mismo, se apalanca a través de impresiones publicitarias escritas, pero hoy en día, sobre todo, mediante una estrategia

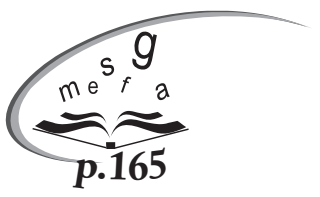
de propuesta de mercadeo digital, a través de su página web. Con este modelo de negocios la infraestructura tecnológica se convierte en un factor fundamental en la organización, la que se ha visto afectada en varias ocasiones por problemas que previamente pudieron ser detectados, y que causaron caídas del sistema y consecuentemente, pérdida en ventas.

\footnotetext{
1 Nombre ficticio en procura de proteger la confidencialidad del nombre real de la organización del estudio.

2 Retail es el término inglés para comercio al por menor o al detalle. Engloba el sector de negocios que va desde supermercados, pasando por tiendas de marca, grandes superficies, centros comerciales, hasta sucursales bancarias y en algunos casos restaurantes (ej. comida rápida). Está muy relacionado con las cadenas de tiendas, franquicias, centrales de compras y hasta hay quien considera que el comercio online podría ir en el mismo saco, aunque es preferible llamarlo como e-retail.
} 
A partir del año 2009 se comenzaron a documentar los incidentes que afectaban el normal funcionamiento de la infraestructura tecnológica en Mecanizados y Eléctricos S.A. y que provocaban caídas o afectación de los principales servicios de la organización. Con los datos recolectados se estableció el indicador «Medición de servicios» (Tabla 1).

Tabla 1. Indicador de medición de servicios a 2011

\begin{tabular}{|l|c|c|c|}
\hline \multirow{2}{*}{ Descripción del servicio } & \multicolumn{3}{|c|}{ Número de caídas anuales } \\
\cline { 2 - 4 } & $\mathbf{2 0 0 9}$ & $\mathbf{2 0 1 0}$ & $\mathbf{2 0 1 1}$ \\
\hline Canales de Intranet. & 15 & 33 & 6 \\
\hline - Canal de Internet. & 3 & 7 & 0 \\
\hline - Servicio de correo. & 6 & 7 & 0 \\
\hline Servidor de operaciones principal. & 3 & 2 & 2 \\
\hline Sistema de operaciones principal. & 8 & 8 & 5 \\
\hline $\begin{array}{l}\text { Copias de seguridad de sistema } \\
\text { de operaciones. }\end{array}$ & 5 & 21 & 0 \\
\hline $\begin{array}{l}\text { Copias de seguridad a equipos de } \\
\text { soporte. }\end{array}$ & 143 & 105 & 1 \\
\hline Servicios de soporte. & 19 & 66 & 4 \\
\hline TOTALIZADO & $\mathbf{2 0 2}$ & $\mathbf{2 4 9}$ & $\mathbf{1 8}$ \\
\hline
\end{tabular}

Fuente. Mecanizados y Eléctricos S.A., (2015).

De lo anterior, se resume que el número de caídas de servicio es significativo, afectando principalmente los relacionados con los servidores de soporte que proporcionan el mecanismo de contingencia a todas las sucursales.

Los resultados anteriores, por sí solos, no proporcionan una imagen global del impacto que producen; sin embargo, si se agrega a los mismos el hecho de que el promedio de clientes potenciales totalizado diariamente por todos los almacenes, entre semana lunes a viernes-, es de 4800 personas y en fines de semana -sábados, domingos y festivos- es de 9400 personas, el indicador de "Medición de servicios» constituye un factor de gran importancia.
La organización ha establecido diversos mecanismos de contingencia, entre los que se destacan el diseño de nuevos esquemas de copias de seguridad y la distribución de la información a equipos de soporte, los cuales se consideran una parte del problema pero no engloban la infraestructura tecnológica de toda la organización.

$$
\text { Por todo esto, }
$$
Mecanizados y Eléctricos S.A. debe buscar la implementación efectiva de un sistema de monitoreo de infraestructura

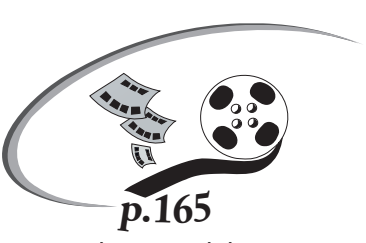
tecnológica centralizada, que permita emitir alertas tempranas de incidentes que atenten contra el sistema actual de tecnología. 


\section{Arquitectura de red actual}

M

ecanizados y Eléctricos S.A. dispone de

un esquema de negocios centralizado basado en el esquema de Cliente/Servidor, en donde todas las operaciones solicitadas por los equipos Cliente se procesan de forma centralizada en equipos Servidores, los cuales almacenan y/o procesan los datos según las operaciones solicitadas.

De igual manera, Mecanizados y Eléctricos S.A., ha implementado la arquitectura de red buscando que todas las sucursales con las que hoy cuenta -Norte, Salitre, Pepe Sierra, Plaza de las Américas y Héroes-, se encuentren interconectadas con la central administrativa en donde se realiza todo el procesamiento de la información.

Internamente los servidores de aplicacio-nes contienen un sistema ERP -Enterprise resource planning/planificación de recursos empresariales- en donde se integran las operaciones de logística, ventas, compras, inventarios, talento humano y tesorería (Figura 1).

Figura 1. Diagrama de distribución lógica de Red

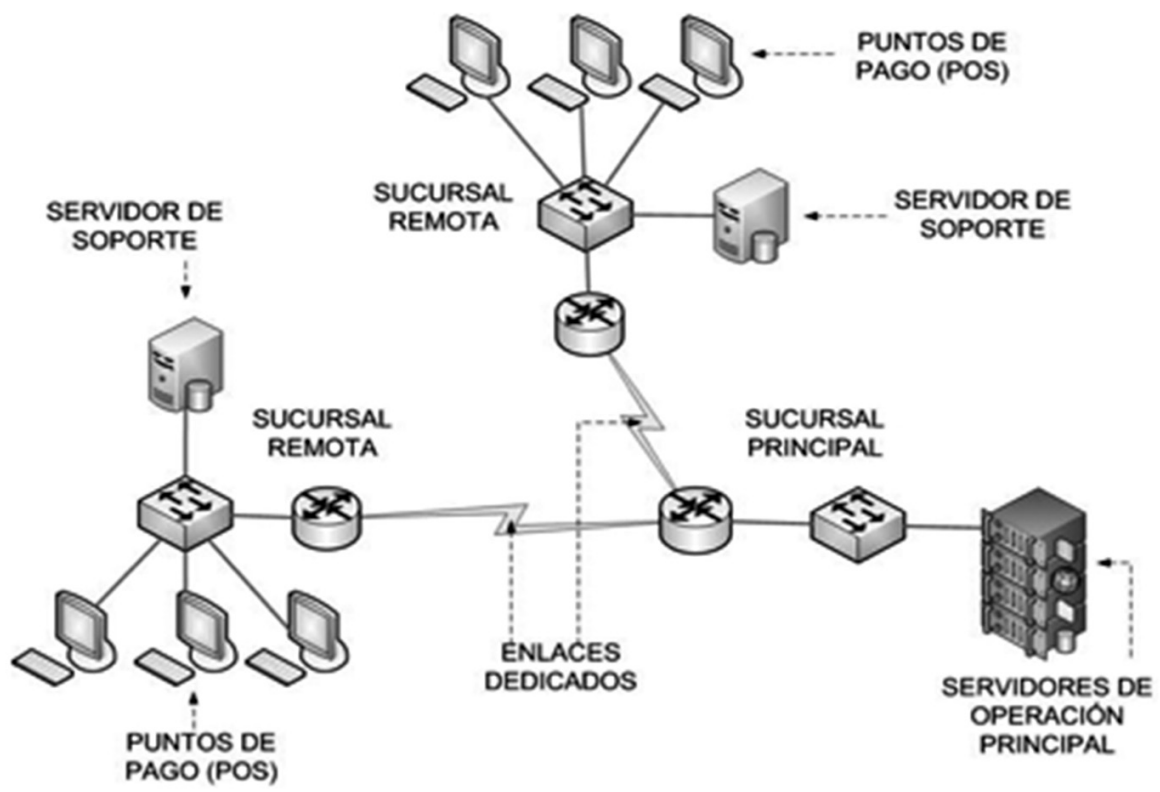

Fuente. Mecanizados y Eléctricos S.A., (2015).

Este sistema centralizado permite que las operaciones de un área en particular se vean reflejadas inmediatamente hacia las otras. Por ejemplo, la venta de un producto incidirá no solamente en el área comercial, sino que al mismo tiempo podrá verse reflejada en tiempo real en las áreas de inventarios, logística y contabilidad, permitiendo la toma de decisiones sin la necesidad de realizar procesos de consolidación de datos posterior (Figura 2). 
Figura 2. Diagrama General de Red Mecanizados y Eléctricos S.A.

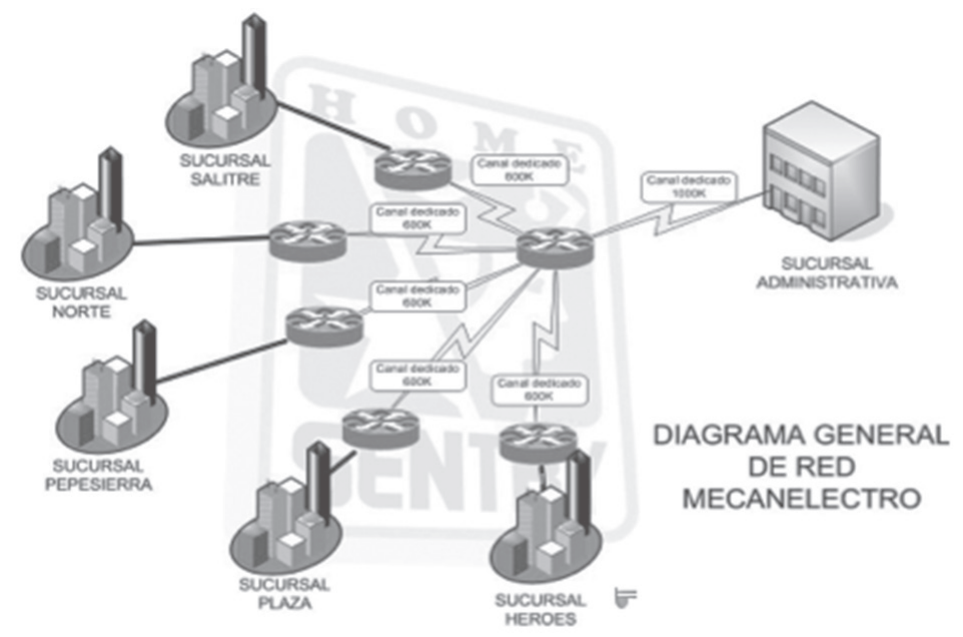

Fuente. Información suministrada por el retail, (2015).

Independiente a los servidores de aplicaciones, se encuentran los servidores web, baser de datos, archivos, mensajería interna, correo corporativo, VPN y Proxy; cada uno de los cuales soportan diferentes servicios a lo largo de toda la organización.

\subsection{Equipos computacionales}

Uno de los elementos que constituye la parte visible de la tecnología computacional en retail está definido por los equipos de cómputo, los cuales son entidades físicas que permiten realizar funciones por medio del hardware y software que lo componen. Existen variedad de equipos de cómputo con funciones bien definidas, los cuales se encuentran subdivididos en las siguientes categorías:

- Equipos de escritorio. Empleados para realizar el procesamiento de información de carácter general, utilizado ampliamente por el usuario final. En esta categoría se pueden destacar los equipos personales y equipos portátiles.
- Servidores. Se encuentran en un punto centralizado de la red organizacional, pudiendo ser accedidos por cualquier usuario en la red. Dentro de los servicios que pueden ser provistos en este tipo de equipos, se encuentran: el servicio web, los servidores de correo que permiten la transmisión de correo electrónico, servidores de aplicaciones específicas, entre otros.

- Equipos activos. Forman parte de la infraestructura de red y activan la interconexión de los computadores que la componen permitiendo que estos puedan compartir información. Entre los principales equipos activos están:

- Switches o conmutadores. Logran interconectar múltiples equipos en una red LAN de forma centralizada, permitiendo redireccionar los datos desde un equipo cliente a un equipo destino.

- Routers o enrutadores. Equipos especializados que permiten que un computador pueda comunicarse con otro en cualquier parte del mundo por medio de las direcciones IP y tablas de enrutamiento. 


\subsection{Práctica versus experiencia en TIC}

La experiencia de retail, está enfocada desde la organización: TIC como experiencia.

\begin{tabular}{|l|}
\hline \multicolumn{1}{|c|}{ TIC en la práctica } \\
\hline - Contextualizado. Uso del servidor para corroborar precios. \\
- Normatividad. El código de barras es la norma. \\
- Recursividad. Adapta la tecnología a las circunstancias. \\
\hline TIC en la experiencia \\
\hline - Sentido de responsabilidad. Compromiso como empresa. \\
- Solidaridad. Se preocupa por los clientes. \\
- Reconstrucción creativa. \\
- Fidelidad. La tecnología fideliza al cliente a través de los servicios. \\
- El compromiso. Ser cliente incluye identificación con la empresa.
\end{tabular}

Diferencias. Se percibe diferencia entre TIC en la parte práctica -normatividad- con relación a las TIC, como experiencia -compromiso, lealtad, sentido-, cuyo énfasis va hacia el cliente como individuo.

\subsection{Necesidades y restricciones}

Con el fin de determinar que la implementación sea satisfactoria, diversas necesidades y restricciones se han establecido para cumplir el sistema de monitoreo en la organización, donde este serállevado a cabo. Los lineamientos establecidos por la empresa son los siguientes:

\section{Restricciones}

- Monitorear todos los servidores corporativos de la empresa permitiendo el monitoreo de sistemas de ficheros, rendimiento, conectividad y registros generados por aplicativos empresariales.

- Generar los diagramas de distribución física y lógica de la infraestructura de red corporativa.

- Generar reportes de rendimiento, calidad de servicio y tiempo de respuesta a incidentes de los sistemas que se encuentren siendo monitoreados.

\section{Acciones}

- No se realizará ningún cambio de configuración de aplicativos existentes sobre equipos servidores y/o de red existentes sin la debida autorización y supervisión por parte del administrador de red de la empresa.

- Los parámetros y objetivos de monitoreo se deben establecer en conjunto con el administrador de red de la empresa.

- La solución de software de monitoreo ha de ser de fácil acceso y administración.

- Evitar realizar la instalación de ningún tipo de aplicativo de software o hardware en servidores y/o equipos de red, que formen parte de los equipos de misión crítica de la empresa. 


\subsection{Los colaboradores y las TIC en las organizaciones}

Hoy en día, las organizaciones buscan ser más competitivas para asegurar su existencia. Hay un incremento del poder en los clientes y una proliferación de productos y servicios personalizados que son desarrollados de acuerdo con la capacidad de innovación en las organizaciones, utilizando, muchas veces, nuevas Tecnologías de la Información y la Comunicación (TIC).

Las organizaciones deben utilizar estrategias de colaboración entre sus trabajadores y las entidades que forman parte de su red.
Para lograrlo, se busca la reformulación y automatización de los procesos a fin de reducir los niveles de la organización y hacer más ágil la toma de decisiones. De este modo, se dispone de una estructura organizacional sencilla y que centra su atención en tres

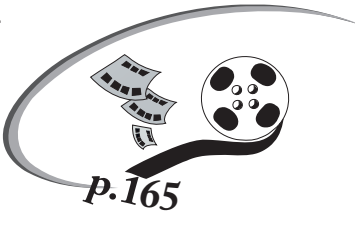
aspectos: el capital humano, el uso de las TIC y las estrategias innovadoras basadas en la colaboración.

Los beneficios de adquirir tecnologías de información se dan cuando se obtiene conciencia de que las ventajas competitivas y la sostenibilidad de los negocios, se aseguran con el adecuado uso de la tecnología (Figura 3).

Figura 3. Los trabajadores como factor de éxito

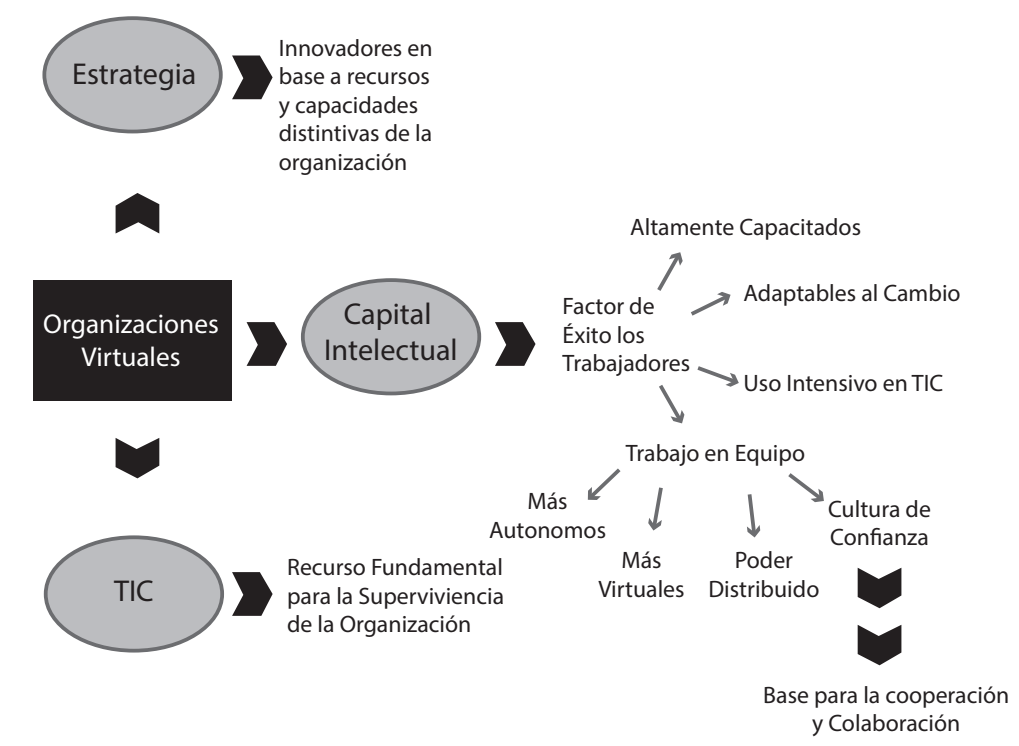

Fuente. Información suministrada por Bao et al., (2010).

\subsection{Soluciones de monitoreo disponibles}

En la actualidad existen diferentes empresas que proporcionan el servicio de monitoreo computacional para diferentes organizaciones; cada una cuenta con características bien definidas y particulares que permiten su aplicación en ambientes heterogéneos. Entre los principales productos que participan en este mercado se encuentran, según información suministrada en el retail:

- ZENOSS Enterprise. Sistema de monitoreo que se distribuye bajo licencia de código abierto, dependiendo del soporte requerido. Una de las principales 
características que tiene Zenoss radica en la capacidad de poder realizar monitoreo de nuevas tecnologías basadas en virtualización y computación en nube, bajo el conjunto de tecnologías de la empresa VMWare la cual se especializa en ambientes virtualizados.

- HP OpenView. Producto desarrollado por Hewlett Packard que consiste en la administración de sistemas y redes de gran escala, en una infraestructura de tecnologías de la información. Una de las principales características de HP OpenView es la amplia integración que posee con equipos de red y software de diversa índole, por lo cual se pueden encontrar más de 60 versiones diferentes para diversas actividades.

- CiscoWorks LAN Management Solution. Producto desarrollado por Cisco System, con el fin de proporcionar un mayor grado de facilidad en la administración de redes basadas en equipos de la misma marca Cisco. Una de las principales ventajas de este producto es que al estar estrechamente relacionado con los productos de la marca se puede obtener un mayor nivel de detalle acerca de problemas, errores, alertas y notificaciones que serían difíciles de obtener con herramientas externas.

- Nagios XI. Producto desarrollado bajo licencia de software libre con soporte comercial, ampliamente utilizado por su versatilidad y alto nivel de personalización a nivel de configuración, que puede ser desarrollado en la plataforma. En la actualidad cuenta con una amplia variedad de complementos desarrollados que permiten implementar funcionalidades específicas sobre plataformas diferentes a Linux.
- Pandora FMS. Producto desarrollado bajo licencia de software libre, que sirve para monitorizar sistemas y aplicativos. Una de las características más importantes, es su versatilidad en el proceso de configuración de plataforma, la que se realiza por medio de la interfaz web incluida en el producto. Una de las principales ventajas de este producto radica en la integración con los complementos desarrollados para Nagios, que le proporciona acceso a una gran variedad de mecanismos de monitoreo implementados en esta última plataforma.

\subsection{Criterios de selección y evaluación}

Es necesario realizar diferenciación entre las diversas características que cada uno posee, con el fin de determinar cuál es el software de monitoreo de red adecuado de acuerdo con las necesidades de la organización.

El proceso de evaluación de las herramientas de monitoreo se debe establecer bajo tres grupos globales que contemplan los aspectos técnicos, financieros y de servicio. Este proceso debe contemplar los aspectos técnicos y financieros, y posteriormente realizar un segundo proceso de selección, en donde se consideren aspectos específicos de servicio, que permitan definir claramente la selección de la herramienta adecuada.

\subsection{Artefacto a diseñar}

El artefacto a diseñar lo constituye un sistema de monitoreo de infraestructura tecnológica centralizada, que permita emitir alertas tempranas de incidentes que atenten contra el sistema actual de tecnología. 


\subsection{Hipótesis propuestas}

H1. La facilidad de uso percibido tendrá un efecto positivo sobre la utilidad percibida del sistema de monitoreo de infraestructura tecnológica.

H2. La utilidad percibida y las normas subjetivas tendrán un impacto positivo sobre la intención del comportamiento de los consumidores.

\subsection{Verificación de la epistemología interpretativa}

Partiendo del modelo pragmático, podría argumentarse una extensión del diseño para darle un mayor valor agregado al sistema de monitoreo, tomando lo modelado desde el punto de vista de epistemología positiva y con ello, se puede plantear una nueva alternativa de extensión a la anterior, trabajando el interrogante específico de un nuevo modelo basado en averiguar y/o corroborar la utilidad del sistema, a través de un trabajo de focus group, al que especificamente se le pregunta sobre cuál considera que es la utilidad del sistema y con base en las respuestas permite rediseñar el modelo original, conformando una nueva alternativa o nueva propuesta. El enfoque pragmático implica desarrollar y profundizar en los detalles de la estrategia del juego enfatizando en sus posibilidades de uso.

Desde el punto de vista interpretativo, se puede utilizar el modelo de aceptación tecnológica denominado TAM. Igualmente, a través de la aplicación de entrevistas a profundidad sobre expectativas de desempeño del sistema propuesto a grupos puntuales 0 focus group se podría instrumentalizar su uso. Por esta ruta se podría incurrir en incrementos de tiempo, costos, y sobre todo, con un alto riesgo en razón a que al final los resultados podrían ser invalidados.

\section{Conclusiones}

T as TIC, logran flexibilidad en las orgaUnizaciones $y$, generalmente, constituyen un primer paso en la mejora de la organización.

La importancia de las TIC en un ámbito más estratégico, radica en tener la capacidad de poder utilizarlas para identificar cuáles son las principales necesidades de los clientes. Es decir, ser una organización donde el cliente es el centro y esta, a su vez, trabaja en función de sus necesidades. Lograrlo pasa por un cambio de cultura organizacional y la necesidad de manejar adecuadamente los cambios que se tengan que realizar en su estructura.
En cuanto a la manera en que se debe realizar la implementación de un sistema de monitoreo centralizado de infraestructura tecnológica en la empresa Mecanizados y Eléctricos S.A., se debe elegir una herramienta que no sólo se ajuste a las necesidades y restricciones de la compañía, sino que también sea configurada, instalada y probada en su totalidad para el uso y funcionamiento correcto en la organización.

No todas las herramientas disponibles serán soluciones apropiadas para todos los ambientes de trabajo, debido a que muchos de estos sistemas son construidos con base a una funcionalidad generalizada, lo cual 
puede proporcionar una falsa perspectiva sin la evaluación adecuada. Por tanto, se debe realizar un proceso de selección y evaluación de cada una de las características que poseen las herramientas y elegir aquella que se ajuste de mejor manera a lo requerido por la empresa.

Con relación a los diseños durante el proceso de definición de elementos, se debe analizar todo el espectro de variables que deberían ejecutarse cuando los parámetros se encuentren fuera de los rangos normales establecidos, lo que contribuirá a aumentar el grado de confiabilidad del sistema. Adicionalmente, se debe contar con la colaboración de personal con amplios conocimientos en el área de tecnología de la compañía, puesto que si no se realiza la adecuada definición de los diferentes parámetros en el sistema de monitoreo a implantar, se pueden presentar inconsistencias que crearían desconfianza sobre el proyecto ejecutado.

\section{Referencias bibliográficas}

McCarthy, J. \& Wright, P. (2004). Technology as Experience. Magazine Interactions. 11(5).

MCao, Q Zhang, J Seydel (2005). B2C e-commerce web site quality: an empirical examination. Recuperado de: Emeraldinsight.com.

Manenti, P. (2010). Key Strategic Challenges in $B 2 B$ e-Commerce. Manufacturing Insights.

Manufacturing executive. (2010).Internet Is Engine of Manufacture's Growth, Study Says. Recuperado de: Ichainnel. com/en/news/21083_570801-internetis-engine-of-manufacturers-growthstudy-says/

Orlikowski, W. (2000). Using Technology and Constituting Structures: A Practice Lens for Studying Technology in Organizations. (s.c) (s.e).

Oracle (2011). Reinventing the Web Channel to Maximize B2B Sales and Customer Satisfaction. (s.c) (s.e).
Stallings, W. (1997). Data and Computer Communications. Quinta edición. Prentice Hall.

Sheldon, P. (2015). The Forrester Wave: B2C e-Commerce Platforms. Forrester Research, Inc., 60 Acorn Park Drive, Cambridge

Tanenbaum, A. S. (1997). Redes de computadoras. Tercera edición. Naucalpan de Juárez: Prentice Hall Hispanoamérica, S.A.

Walker, B. (2000). The Art of Production Environment Engineering. Palo Alto: Sun Microsystems, Inc.

Zhang, B. (2004). Ecommerce Insights: Where Technology Meets Business. Recuperado de: http://ecommerce.insightin.com/ network/network_management.html.

Walker, B., Freeman, E., \& MacGowan B. (2010).Trends 2010: e-Commerce Platform and Technology Forrester Research. (s.c) (s.e). 

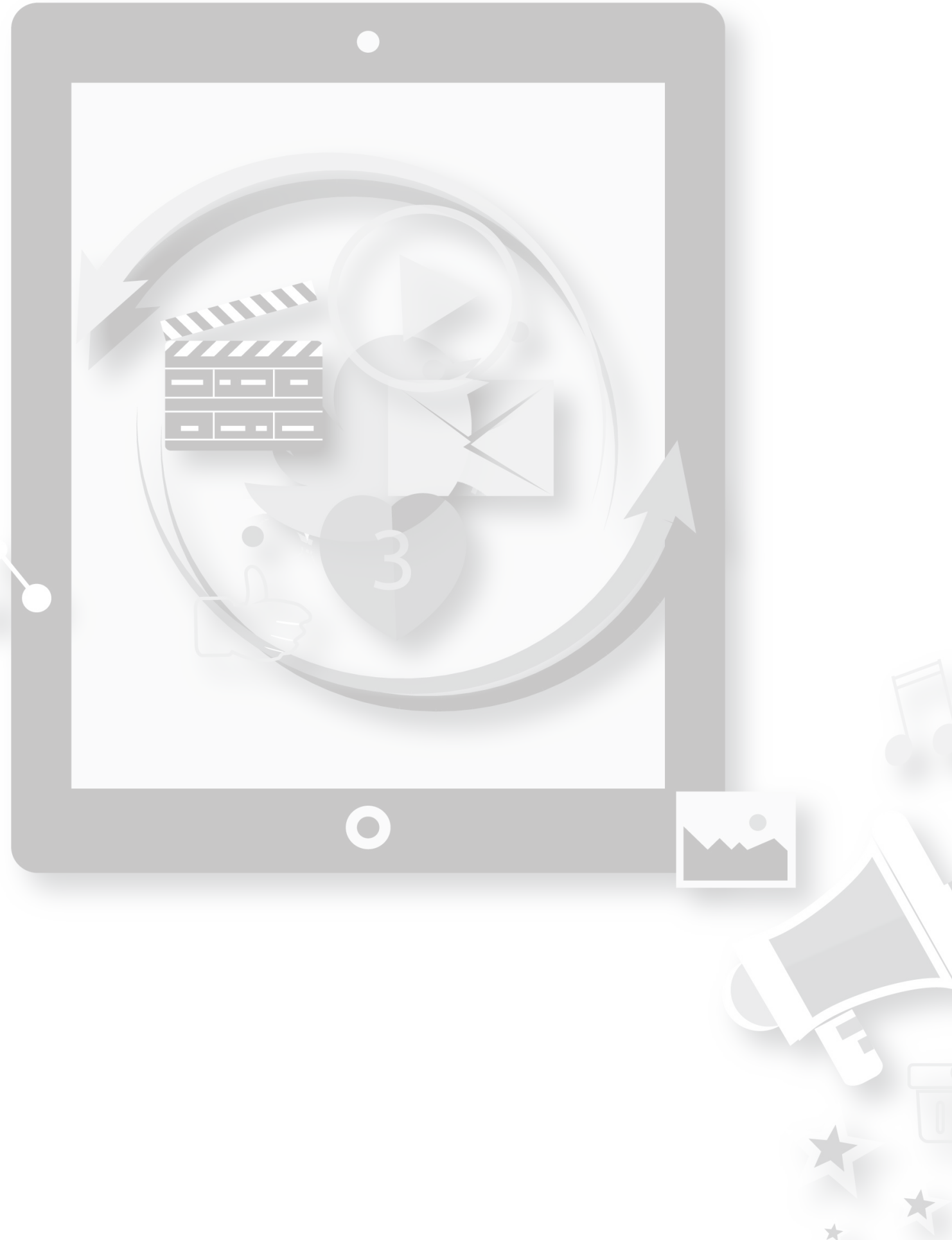